\title{
The prognostic role and reduced expression of FOXJ2 in human hepatocellular carcinoma
}

\author{
ZHONGBAO ZHANG $^{1^{*}}$, GUANGJU MENG ${ }^{2 *}$, LIANG WANG $^{1}$, YINGYING MA $^{3}$ and ZHONGZHENG GUAN ${ }^{1}$ \\ ${ }^{1}$ Department of General Surgery, The Third People's Hospital of Liaocheng, Liaocheng, Shandong 252000; \\ ${ }^{2}$ Department of Infectious Diseases, Tai'an Center Hospital, Tai'an, Shandong 271000; ${ }^{3}$ Department of Surgery, \\ The Third People's Hospital of Liaocheng, Liaocheng, Shandong 252000, P.R. China
}

Received July 1, 2015; Accepted April 29, 2016

DOI: $10.3892 / \mathrm{mmr} .2016 .5261$

\begin{abstract}
The current study aimed to investigate the potential role of the FOXJ2 (forkhead box J2) protein in the pathology of hepatocellular carcinoma (HCC). Western blotting was performed to determine the expression levels of FOXJ2 in HCC tissues and HCC cells. Specimens from 110 patients with HCC undergoing hepatic resection were evaluated for FOXJ2 expression using an immunohistochemical assay. The correlation between FOXJ2 expression and clinicopathological factors of the patients was determined by statistical analysis to determine the prognostic merit of FOXJ2 expression in HCC. The detailed involvement of FOXJ2 in the regulation of HCC proliferation was further investigated using FOXJ2-targeting small interfering RNA (siRNA). FOXJ2 protein was identified to be significantly downregulated in HCC tissues compared with adjacent normal liver tissues. Immunohistochemical analysis demonstrated that the expression of FOXJ2 was negatively correlated with $\mathrm{Ki}-67$ levels in HCC specimens $(\mathrm{r}=-0.679, \mathrm{P}<0.001)$. Furthermore, statistical analysis indicated FOXJ2 expression was significantly associated with histological differentiation $(\mathrm{P}=0.005)$, the size of largest tumor $(\mathrm{P}=0.002)$ and metastasis $(\mathrm{P}=0.036)$. Using Kaplan-Meier analysis, it was demonstrated that high FOXJ2 expression levels predicted significantly improved patient survival rates compared with low FOXJ2 expression levels $(\mathrm{P}<0.001)$. In addition, it was observed that interference of FOXJ2 expression using siRNA oligos led to the promotion of proliferation of HepG2 cells. FOXJ2 was markedly downregulated in HCC tissues. The expression of FOXJ2 was correlated with tumor size, histological differentiation and metastasis. Low expres-
\end{abstract}

Correspondence to: Professor Zhongzheng Guan, Department of General Surgery, The Third People's Hospital of Liaocheng, 62 Weiyu Road, Liaocheng, Shandong 252000, P.R. China

E-mail: guanzhongzheng1a@126.com

*Contributed equally

Key words: FOXJ2, hepatocellular carcinoma, prognostic marker, proliferation sion levels of FOXJ2 predicted poor prognosis for patients with $\mathrm{HCC}$, suggesting that FOXJ2 may be a candidate prognostic marker of HCC. Depletion of FOXJ2 caused the promotion of HCC cell proliferation, implicating that FOXJ2 may serve an inhibitory role in the regulation of HCC cell proliferation.

\section{Introduction}

Hepatocellular carcinoma (HCC) is the fifth most common cancer in Eastern Asia (1). However, it remains to be a type of cancer with a low 5-year survival rate worldwide $(<10 \%)$ when in the advanced stages, due to difficulties in detection and the high frequency of tumor recurrence $(2,3)$. Hepatocarcinogenesis involves complex molecular mechanisms, involving alterations in the structure or expression of several tumor suppressor genes and oncogenes $(4,5)$. However, the detailed molecular mechanisms underlying HCC metastasis remain unclear. Thus, the identification of novel biomarkers to increase specificity or sensitivity for early diagnosis and to improve the prognosis of $\mathrm{HCC}$ is required.

Forkhead box J2 (FoxJ2), a member of the forkhead transcription factor family (6), has been identified in several mammals and vertebrates (7-9) and is widely distributed in different organs and tissues, in adults and in the fetus. FOXJ2 has been suggested to be involved in positively regulating the progression of the cell cycle or aiding in tumorigenesis (10). Wang et al (11) reported that overexpression of FOXJ2 was able to reduce the migration of breast cancer cells, and inhibit the metastasis of human breast cancer by regulating the epithelial-mesenchymal transition (EMT) key markers E-cadherin and vimentin. In human glioma cells, overexpression of FOXJ2 has been reported to increase E-cadherin expression and reduce vimentin expression (12). Overexpression of FOXJ2 has been observed to significantly inhibit cell migration, and knockdown of FOXJ2 to promote cellular motility, thus it was suggested that FOXJ2 suppresses cell migration and invasion in glioma (12).

The current study aimed to investigate the potential involvement of FOXJ2 in HCC pathology and to evaluate the prognostic value of FOXJ2 expression in HCC. It was identified that FOXJ2 was significantly downregulated in HCC specimens, compared with adjacent nontumorous tissues. Furthermore, it was observed that the expression of FOXJ2 
was correlated with histological differentiation, the size of the largest tumor and metastasis, and Ki-67 expression levels. In vitro, knockdown of FOXJ2 expression was able to promote HepG2 cell proliferation. These observations may provide novel insight into the mechanisms underlying HCC development.

\section{Materials and methods}

Patients and tissue samples. All the HCC samples used in the current study were from 110 patients who underwent hepatic resection without preoperative systemic chemotherapy at The Third People's Hospital of Liaocheng (Liaocheng, China). The main clinical and pathological characteristics are presented in Table I.

Immunohistochemistry. Tissues were formalin-fixed and paraffin-embedded for immunohistochemical study. The sections were dewaxed in xylene and rehydrated in graded ethanol. Thereafter, the sections were processed in $10 \mathrm{mmol} / \mathrm{l}$ citrate buffer ( $\mathrm{pH}$ 6.0) and heated to $105^{\circ} \mathrm{C}$ in an autoclave for three cycles of 5 min each for antigen retrieval. Subsequently, endogenous peroxidase activity was blocked by soaking in $0.3 \%$ hydrogen peroxide for $15 \mathrm{~min}$ subsequent to resting of the sections room temperature for $1 \mathrm{~h}$. Goat serum (Wuhan Huamei Biotech Co., Ltd., Wuhan, China) was applied to block any nonspecific reactions for $15 \mathrm{~min}$. The sections were then incubated overnight at $4^{\circ} \mathrm{C}$ with goat anti-human FOXJ2 polyclonal antibody (1:150; sc-54374; Santa Cruz Biotechnology, Inc., Dallas, TX, USA), and anti-Ki67 mouse monoclonal antibody (1:100; sc-23900; Santa Cruz Biotechnology, Inc.). Negative control slides were processed in parallel using a nonspecific immunoglobulin IgG (Sigma-Aldrich, St. Louis, MO, USA) at the same concentration as the primary antibody. All slides were processed using the peroxidase-antiperoxidase method (Dako, Hamburg, Germany). Subsequent to rinsing in water, the sections were counterstained with hematoxylin, dehydrated and coverslipped. All of the immunostained sections were evaluated in a blinded manner without knowledge of the clinical and pathological features of the patients. For assessment of FOXJ2, five high-power fields in each specimen were selected randomly, and nuclear (cytoplasmic) staining was examined. Greater than 500 cells were counted to determine the labeling index, which represented the percentage of immunostained cells relative to the total number of cells. In half of the samples, staining was repeated twice to avoid possible technical errors, however similar results were obtained in these samples. A second investigator using a multihead microscope assessed the obtained results, and a consensus was achieved.

Cell culture. One normal hepatocyte cell lines (L02) and 3 HCC cell lines (HepG2, Huh7 and Hep1) were obtained from the Institute of Cell Biology of the Chinese Academy of Sciences (Shanghai). Cells were cultured in Roswell Park Memorial Institute 1640 and Dulbecco's modified Eagle's medium (DMEM) supplemented with $10 \%$ fetal bovine serum (FBS), and $100 \mathrm{U} / \mathrm{ml}$ penicillin-streptomycin mixture (all mediums from Invitrogen; Thermo Fisher Scientific, Inc., Waltham, MA, USA) in $5 \% \mathrm{CO}_{2}$ at $37^{\circ} \mathrm{C}$.
The sequences of the primers for FOXJ2 were as follows: 5'-AAGCTTGGAAGTGCCTCCCAG-3', and the control 5'-TGATGACATCAAGAAGGTGGTGAAG-3'. In addition, a mock group with no transfection was also used as a control. Cells were seeded the day prior to transfection using DMEM with $10 \%$ FBS however without antibiotics. Transfection was performed using Lipofectamine 2000 transfection reagent (Invitrogen; Thermo Fisher Scientific, Inc.) according to the manufacturer's protocol. The cells were incubated with the control vectors and Lipofectamine plus reagent complexes for 4-6 h at $37^{\circ} \mathrm{C}$, and FBS was added to DMEM at a final concentration of $10 \%$. The cells were used for subsequent experiments at $48 \mathrm{~h}$ subsequent to transfection.

Western blot analysis. Tissue and cell proteins were homogenized in a homogenization buffer containing $1 \mathrm{M}$ Tris $\mathrm{HCl}(\mathrm{pH} 7.5), 1 \%$ Triton X-100, $1 \%$ Nonidet P-40, 10\% sodium dodecyl sulfate (SDS), $0.5 \%$ sodium deoxycholate, $0.5 \mathrm{M}$ ethylenediaminetetraacetic acid, leupeptin $10 \mu \mathrm{g} / \mathrm{ml}$, aprotinin $10 \mu \mathrm{g} / \mathrm{ml}$ and $1 \mathrm{mM}$ phenylmethylsulfonyl fluoride, then were centrifuged at $10,000 \mathrm{xg}$ for $30 \mathrm{~min}$ at $4^{\circ} \mathrm{C}$ to collect the supernatant liquid. Protein concentrations were determined with a Bio-Rad protein assay (Bio-Rad Laboratories, Inc., Hercules, CA, USA). The total cellular protein extracts were separated by 4-15\% SDS-polyacrylamide gel (Bio-Rad Laboratories, Inc.) electrophoresis and transferred to a nitrocellulose membrane. Membranes were blocked with 5\% non-fat dry milk in phosphate-buffered saline (PBS) for $2 \mathrm{~h}$ at room temperature, then were incubated with antibodies against anti-FOXJ2 (1:1,000; cat. no. sc-54374; Santa Cruz Biotechnology, Inc.) and GAPDH (1:1,000; cat. no. G5262; Sigma-Aldrich) in PBS with the milk about $1 \mathrm{~h}$ at the room temperature. Blots were washed three times in PBS buffer, followed by incubation with the horseradish peroxidase-linked secondary antibodies (1:500; cat. no. sc-51625; Santa Cruz Biotechnology, Inc.). The specific proteins in the blots were visualized using the enhanced chemiluminescence reagent (NEN Life Science Products, Inc., Boston, MA, USA). The experiments were conducted on three separate occasions.

Cell proliferation assay. Cell proliferation was determined using the Cell Counting Kit-8 (CCK-8) assay following the manufacturer's instructions (Dojindo Molecular Technologies, Inc., Kumamoto, Japan). In brief, cells that were transfected with small interfering RNA (siRNA) were seeded at a density of $2 \times 10^{4} /$ well into a 96 -well cell culture cluster (Corning Incorporated, Corning, NY, USA) in $100 \mu 1$ culture medium and incubated overnight. CCK- 8 reagents were added to a subset of the wells, and they were incubated for $2 \mathrm{~h}$ at $37^{\circ} \mathrm{C}$. The absorbance was recorded with an automated plate reader. Each experiment was performed in triplicate and repeated a minimum of three times.

Statistical analysis. Statistical analysis was performed using the SPSS 17.0 software package (SPSS, Inc, Chicago, IL, USA). The association between FOXJ2 and Ki-67 expression and clinicopathological features was analyzed using the $\chi^{2}$ test. FOXJ2 and Ki67 expression was studied using the Spearman rank correlation test due to the nonparametric nature of the data. For analysis of survival data, Kaplan-Meier curves were constructed, and 
A
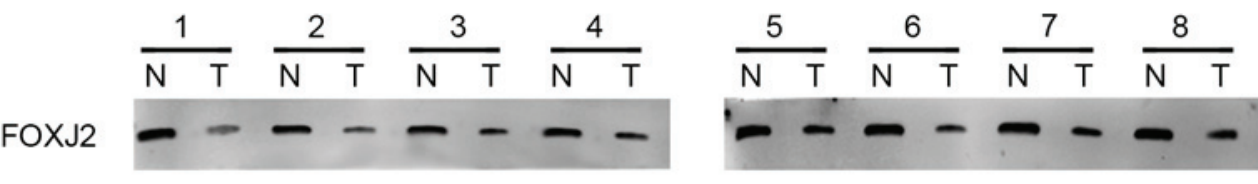

GAPDH

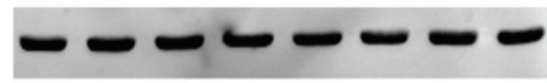

B

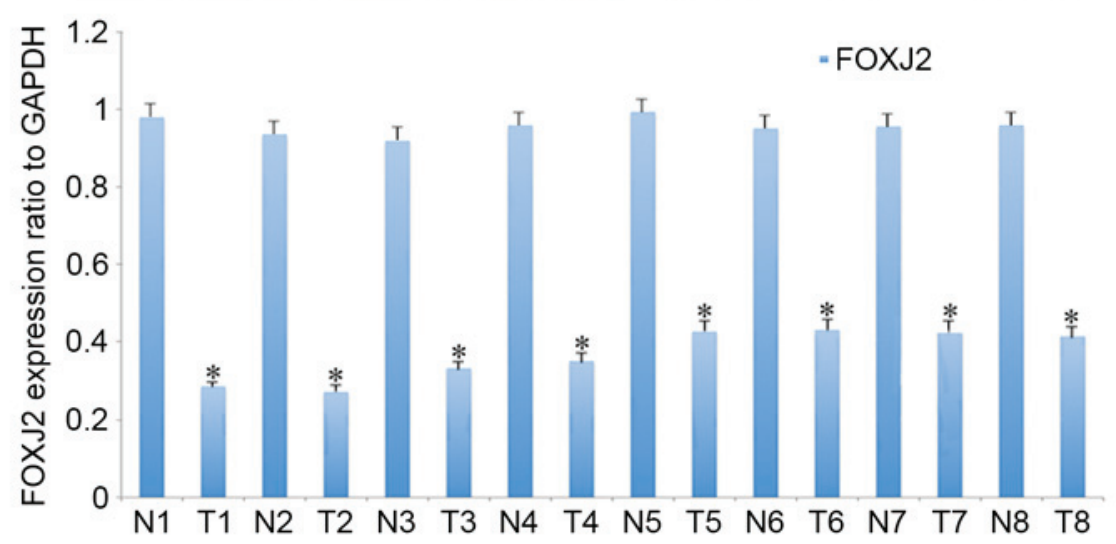

C
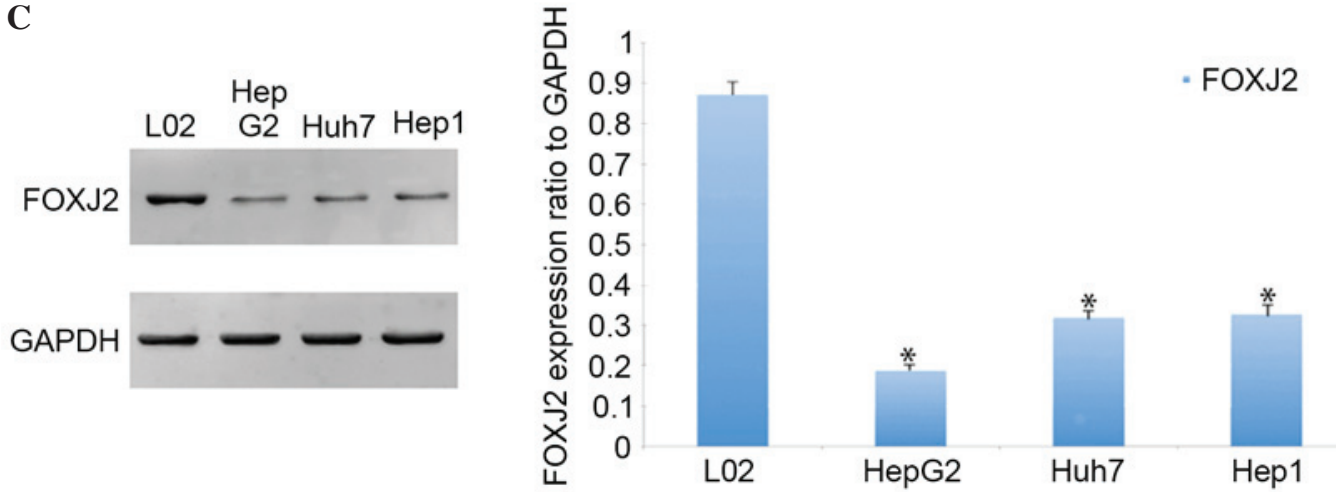

Figure 1. FOXJ2 was downregulated in human HCC tissues and HCC cell lines. (A) Western blot analysis was performed to detect the expression of FOXJ2 in 8 representative paired HCC tumor tissues (T) and adjacent non-tumor tissues (N). GAPDH was used as a loading control. (B) The band intensity of FOXJ2 and GAPDH was quantified and the FOXJ2 protein level was normalized to the GAPDH protein. The data are presented as the mean \pm standard deviation of three independent experiments ("P $<0.005$, vs. adjacent non-tumor tissues). (C) Western blot analysis of endogenous FOXJ2 expression in one normal hepatocyte cell line (L02) and three human HCC cell lines (HepG2, Huh7 and Hep1). GAPDH was used as a loading control ("P<0.05, vs. L02 cells). Experiments were repeated a minimum of three times. FOXJ2, forkhead box J2; HCC, hepatocellular carcinoma; GAPDH, glyceraldehyde 3-phosphate dehydrogenase.

the log-rank test was performed. Multivariate analysis was performed using Cox's proportional hazards model, with $\mathrm{P}<0.05$ considered to indicate a statistically significant difference. The results were expressed as the mean \pm standard deviation.

\section{Results}

FOXJ2 expression in HCC and HCC tissues. To investigate whether FOXJ2 served a role in the development and progression of $\mathrm{HCC}$, its expression pattern was assessed in HCC and adjacent normal tissues. Using western blot analysis, significantly lower expression levels of FOXJ2 were identified in HCC tissues compared with the matched normal tissues (Fig. 1A and B). Furthermore, samples collected from 110 patients with HCC who underwent hepatic resection without preoperative systemic chemotherapy at the Third People's Hospital of Liaocheng. These samples were used to prepare a tissue microarray in order to examine the expression of FOXJ2 and the proliferation marker Ki-67. Immunohistochemistry was conducted to determine the expression of FOXJ2 and Ki-67 in HCC tissues and adjacent normal tissues. The expression rate of FOXJ2 and Ki-67 in each tumorous specimen were counted and summarized. Coinciding with the western blot results, immunohistochemical analysis indicated that the expression of FOXJ2 was significantly lower in HCC specimens compared with the nontumorous samples, with the inverse observed for Ki67 expression (Fig. 2). Notably, the expression rate of FOXJ2 was significantly correlated with Ki67 expression in HCC specimens with a co-efficient of -0.679 (Fig. 3). These observations indicated that FOXJ2 was evidently downregulated in HCC specimens.

The correlation between FOXJ2 expression and clinicopathologic variables in HCC specimens. In order to further tackle the involvement of FOXJ2 expression in HCC progression, the correlation between FOXJ2 expression and clinicopathologic factors was analyzed. According to the expression rate of FOXJ2, the HCC specimens were separated into two groups, the high and low FOXJ2 expression 
Table I. Clinicopathological features of hepatocellular carcinoma in relation to the FOXJ2/Ki67 expression pattern.

\begin{tabular}{|c|c|c|c|c|c|}
\hline \multirow[b]{2}{*}{ Clinicopathological feature } & \multirow[b]{2}{*}{ Total number per group } & \multicolumn{2}{|c|}{ FOXJ2 expression } & \multirow[b]{2}{*}{ P-value } & \multirow[b]{2}{*}{$\chi^{2}$ value } \\
\hline & & Low & High & & \\
\hline All cases & 110 & 63 & 47 & & \\
\hline \multicolumn{6}{|l|}{ Age (years) } \\
\hline$\leq 50$ & 61 & 36 & 25 & 0.680 & 0.170 \\
\hline$>50$ & 49 & 27 & 22 & & \\
\hline \multicolumn{6}{|l|}{ Gender } \\
\hline Male & 89 & 52 & 37 & 0.614 & 0.254 \\
\hline Female & 21 & 11 & 10 & & \\
\hline \multicolumn{6}{|l|}{ Histological differentiation } \\
\hline Well & 11 & 2 & 9 & $0.005^{\mathrm{a}}$ & 10.638 \\
\hline Moderate & 83 & 48 & 35 & & \\
\hline Poor & 16 & 13 & 3 & & \\
\hline \multicolumn{6}{|l|}{ Size of largest tumor $(\mathrm{cm})$} \\
\hline$\leq 5$ & 63 & 28 & 35 & $0.002^{\mathrm{a}}$ & 9.916 \\
\hline$>5$ & 47 & 35 & 12 & & \\
\hline \multicolumn{6}{|l|}{ Tumor number } \\
\hline Solitary & 74 & 41 & 33 & 0.570 & 0.322 \\
\hline Multiple & 36 & 22 & 14 & & \\
\hline \multicolumn{6}{|l|}{$\mathrm{HBV}$} \\
\hline Positive & 88 & 54 & 34 & 0.083 & 3.009 \\
\hline Negative & 22 & 9 & 13 & & \\
\hline \multicolumn{6}{|l|}{ Liver cirrosis } \\
\hline Yes & 89 & 11 & 10 & 0.614 & 0.254 \\
\hline No & 21 & 52 & 37 & & \\
\hline \multicolumn{6}{|l|}{ Microvascular invasion } \\
\hline Yes & 31 & 19 & 12 & 0.594 & 0.285 \\
\hline No & 79 & 44 & 35 & & \\
\hline \multicolumn{6}{|l|}{ Metastasis } \\
\hline Yes & 16 & 50 & 44 & $0.036^{\mathrm{a}}$ & 4.399 \\
\hline No & 94 & 13 & 3 & & \\
\hline \multicolumn{6}{|l|}{ Serum AFP value (ng/ml) } \\
\hline$\leq 50$ & 42 & 20 & 22 & 0.108 & 2.587 \\
\hline$>50$ & 68 & 43 & 25 & & \\
\hline \multicolumn{6}{|l|}{ Ki67 } \\
\hline Low & 59 & 17 & 42 & $<0.001^{\mathrm{a}}$ & 22.848 \\
\hline High & 51 & 38 & 13 & & \\
\hline
\end{tabular}

${ }^{\mathrm{a}} \mathrm{P}<0.05$. FOXJ2, forkhead box J2; HBV, hepatitis B virus; AFP, $\alpha$-fetoprotein.

groups. It was identified that the expression of FOXJ2 was significantly correlated with histological differentiation, size of largest tumor, metastasis and Ki67 expression (Table I). However, there is no correlation between FOXJ2 expression and other clinicopathologic factors, including age, gender, tumor number, hepatitis B virus status, liver cirrosis, microvascular invasion and serum $\alpha$-fetoprotein value. Furthermore, the survival status of the patients was measured and the association between different clinicopathological variables and survival was analyzed. It was identified that the survival status of the patients was significantly correlated with various clinicopathological parameters, including histological differentiation, size of largest tumor, metastasis, FOXJ2 expression and Ki67 expression (Table II). In addition, multivariate analysis using Cox's proportional hazards model demonstrated that the histological differentiation, size of largest tumor, metastasis and FOXJ2 expression were independent prognostic indicators for patient overall survival (Table III). Due to the fact that our observations indicated that FOXJ2 expression was associated with the survival of patients 
Table II. Survival status and clinicopathological parameters in hepatocellular carcinoma specimens.

\begin{tabular}{|c|c|c|c|c|c|}
\hline \multirow[b]{2}{*}{ Parameter } & \multirow[b]{2}{*}{ Total } & \multicolumn{2}{|c|}{ Survival status } & \multirow[b]{2}{*}{ P-value } & \multirow[b]{2}{*}{$\chi^{2}$ value } \\
\hline & & Dead $(n=64)$ & Alive $(n=46)$ & & \\
\hline \multicolumn{6}{|l|}{ Age (years) } \\
\hline$\leq 50$ & 61 & 39 & 22 & 0.770 & 0.085 \\
\hline$>50$ & 49 & 30 & 19 & & \\
\hline \multicolumn{6}{|l|}{ Gender } \\
\hline Male & 89 & 59 & 30 & 0.111 & 2.534 \\
\hline Female & 21 & 10 & 11 & & \\
\hline \multicolumn{6}{|c|}{ Histological differentiation } \\
\hline Well & 11 & 3 & 8 & $0.016^{\mathrm{a}}$ & 8.307 \\
\hline Moderate & 83 & 53 & 30 & & \\
\hline Poor & 16 & 13 & 3 & & \\
\hline \multicolumn{6}{|c|}{ Size of largest tumor $(\mathrm{cm})$} \\
\hline$\leq 5$ & 63 & 33 & 30 & $0.009^{\mathrm{a}}$ & 6.751 \\
\hline$>5$ & 47 & 36 & 11 & & \\
\hline \multicolumn{6}{|c|}{ Tumor number } \\
\hline Solitary & 74 & 43 & 31 & 0.151 & 2.063 \\
\hline Multiple & 36 & 26 & 10 & & \\
\hline \multicolumn{6}{|l|}{$\mathrm{HBV}$} \\
\hline Positive & 88 & 58 & 30 & 0.167 & 1.905 \\
\hline Negative & 22 & 11 & 11 & & \\
\hline \multicolumn{6}{|c|}{ Liver cirrosis } \\
\hline Yes & 89 & 59 & 30 & 0.111 & 2.534 \\
\hline No & 21 & 10 & 11 & & \\
\hline \multicolumn{6}{|c|}{ Microvascular invasion } \\
\hline Yes & 31 & 22 & 9 & 0.263 & 1.254 \\
\hline No & 79 & 47 & 32 & & \\
\hline \multicolumn{6}{|l|}{ Metastasis } \\
\hline Yes & 16 & 14 & 2 & $0.027^{\mathrm{a}}$ & 4.915 \\
\hline No & 94 & 55 & 39 & & \\
\hline \multicolumn{6}{|c|}{ Serum AFP value (ng/ml) } \\
\hline$\leq 50$ & 42 & 25 & 17 & 0.585 & 0.298 \\
\hline$>50$ & 68 & 44 & 24 & & \\
\hline \multicolumn{6}{|l|}{ FOXJ2 } \\
\hline Low & 63 & 55 & 8 & $<0.001^{\mathrm{a}}$ & 38.085 \\
\hline High & 47 & 14 & 33 & & \\
\hline \multicolumn{6}{|l|}{ Ki67 } \\
\hline Low & 59 & 23 & 36 & $<0.001^{\mathrm{a}}$ & 19.278 \\
\hline High & 51 & 41 & 10 & & \\
\hline
\end{tabular}

${ }^{\mathrm{a}} \mathrm{P}<0.05$. FOXJ2, forkhead box J2; HBV, hepatitis B virus; AFP, $\alpha$-fetoprotein.

with HCC, the survival curves of patients with HCC with low and high levels of FOXJ2 expression were assessed. Patients with low expression levels of FOXJ2 had significantly poorer survival, compared with those in the high FOXJ2 expression group (Fig. 4).

FOXJ2 was downregulated in HCC cell lines. $\mathrm{HCC}$ cell lines and a L02 hepatocyte cell line were used to analyze the role of FOXJ2 in HCC cell physiology. The expression levels of FOXJ2 in these cell lines were first determined using western blot analysis. It was identified that $\mathrm{L} 02$ cells had the highest expression of FOXJ2 of all the cell lines examined (Fig. 1C).

Depletion of FOXJ2 led to enhanced cell proliferation. Due to the fact that FOXJ2 expression was observed to be inversely 
Table III. Univariate and multivariate analysis of prognostic factors using Cox proportional hazards model.

\begin{tabular}{|c|c|c|c|c|c|c|}
\hline \multirow[b]{2}{*}{ Factor } & \multicolumn{3}{|c|}{ Univariate analysis } & \multicolumn{3}{|c|}{ Multivariate analysis } \\
\hline & RR & $95 \% \mathrm{CI}$ & P-value & $\mathrm{HR}$ & $95 \% \mathrm{CI}$ & P-value \\
\hline Age $(\leq 50 />50)$ & 0.895 & $0.556-1.440$ & 0.674 & - & - & - \\
\hline Gender $(\mathrm{M} / \mathrm{F})$ & 1.670 & $0.854-3.267$ & 0.134 & - & - & - \\
\hline Differentiation (Well/Moderate/Poor) & 3.557 & $1.116-11.337$ & $0.032^{\mathrm{a}}$ & 1.707 & $0.508-5.735$ & 0.387 \\
\hline Tumor size $(\leq 5 \mathrm{~cm} />5 \mathrm{~cm})$ & 2.263 & $1.404-3.647$ & $0.001^{\mathrm{a}}$ & 1.429 & $0.862-2.369$ & 0.167 \\
\hline Tumor number (Solitary/Multiple) & 1.466 & $0.898-2.392$ & 0.126 & - & - & - \\
\hline $\operatorname{HBV}(+/-)$ & 1.514 & $0.795-2.886$ & 0.207 & - & - & - \\
\hline Liver cirrosis $(+/-)$ & 1.740 & $0.889-3.405$ & 0.106 & - & - & - \\
\hline Microvascular invasion (+/-) & 1.572 & $0.946-2.613$ & 0.081 & - & - & - \\
\hline Metastasis (+/-) & 3.077 & $1.687-5.612$ & $<0.001^{\mathrm{a}}$ & 2.151 & $1.146-4.036$ & $0.017^{\mathrm{a}}$ \\
\hline AFP value $(\leq 50 \mathrm{ng} / \mathrm{ml} />50 \mathrm{ng} / \mathrm{ml})$ & 1.222 & $0.748-1.997$ & 0.424 & - & - & - \\
\hline FOXJ2 (low/high) & 0.171 & $0.094-0.311$ & $<0.001^{\mathrm{a}}$ & 4.692 & $2.490-8.844$ & $<0.001^{\mathrm{a}}$ \\
\hline
\end{tabular}

${ }^{\mathrm{a}} \mathrm{P}<0.05$. CI, confidence interval; HR, hazard ratio; M, male; F, female; HBV, hepatitis B virus; AFP, $\alpha$-fetoprotein; FOXJ2, forkhead box J2.

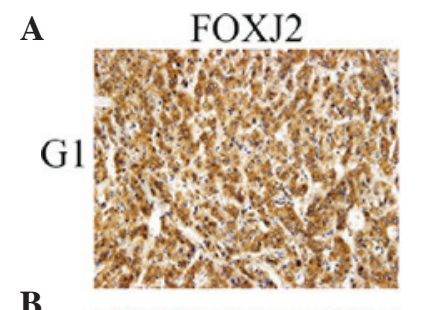

B

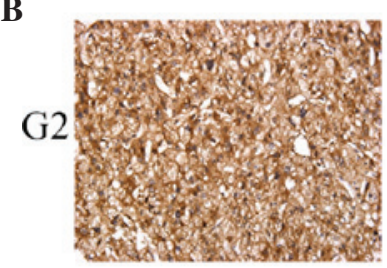

$\mathbf{C}$

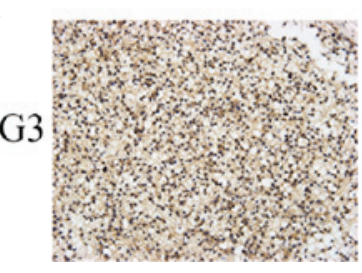

Ki-67
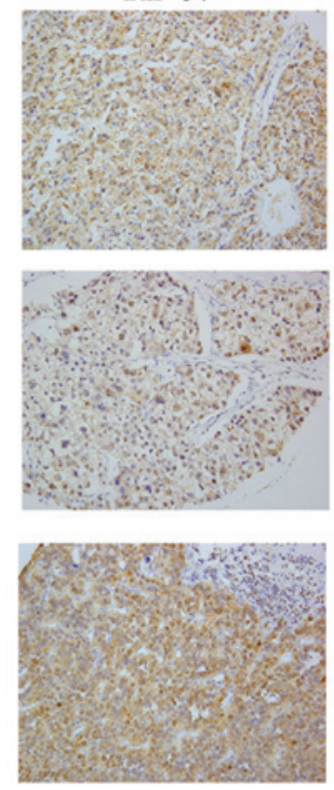
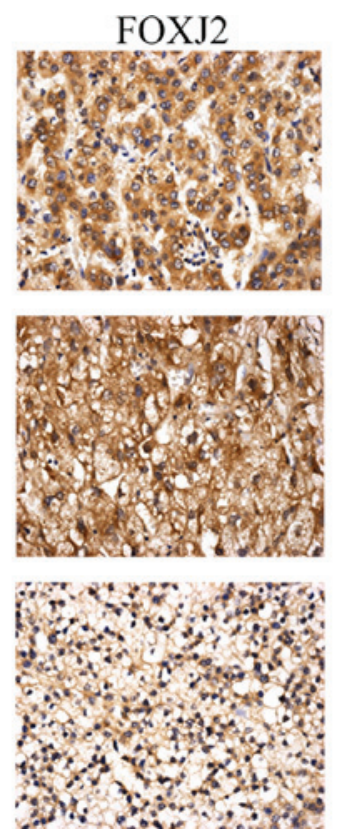

Ki-67
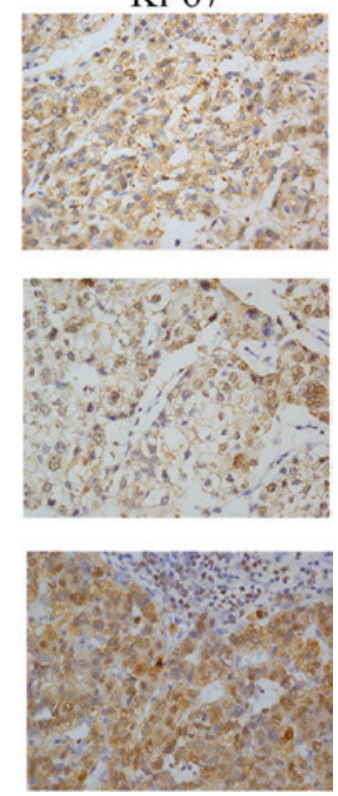

Figure 2. Immunohistochemical analysis of FOXJ2 and Ki-67 expression in HCC and adjacent noncancerous liver tissues. Paraffin-embedded tissue sections were stained with antibodies for FOXJ2 and Ki-67 and counterstained with hematoxylin. (A) FOXJ2 and Ki-67 immunoreactivity in cancer tissue from a tumor classified as G1. (B) FOXJ2 and Ki-67 staining in cancer tissue from a tumor classified as G2. (C) FOXJ2 and Ki-67 staining in cancer tissue from a tumor classified as G3. Left images at a magnification of x200 and right images at a magnification of x400. FOXJ2, forkhead box J2; HCC, hepatocellular carcinoma; G, tumor grade.

correlated with histological differentiation and Ki-67 expression in HCC specimens, it was hypothesized that FOXJ2 may influence the proliferation of HCC cells. Transfection of FOXJ2-siRNA oligos was performed to deplete FOXJ2 expression in three HCC cell lines. Subsequent to transfection of FOXJ2-targeting siRNAs, three HCC cell lines were subjected to western blot analysis to determine the interference efficiencies of different siRNA oligos. As presented in Fig. 5A and B, si-FOXJ2 of the HepG2 cell line was significantly knocked down. Subsequently, the impact of FOXJ2 depletion on HepG2 cell proliferation was determined using the CCK-8 assay. As presented in Fig. 5C, FOXJ2-depleted cells exhibited enhanced cell growth, compared with the mock and control siRNA-transfected cells. The results obtained demonstrated that FOXJ2 depletion could promote the proliferation of hepatocytes.

\section{Discussion}

HCC prognosis remains unsatisfactory due to its high rates of recurrence and metastasis. Despite significant improvements in surveillance and clinical treatment strategies (13), traditional therapeutic methods, including chemotherapy and surgical operation, remain inadequate. Therefore, it is critical to identify patients with poor prognosis for timely interven- 


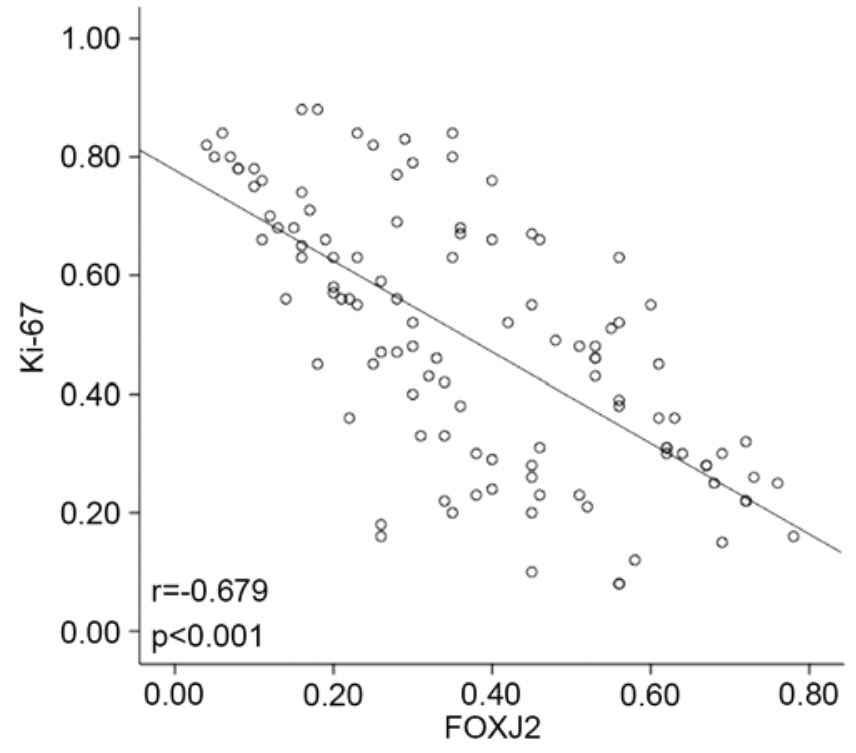

Figure 3. Correlation between FOXJ2 and Ki-67 expression in hepatocellular carcinoma. Scatter plot of FOXJ2 against Ki-67 with the regression line indicating a negative correlation between them using Spearman's rank correlation co-efficient. FOXJ2, forkhead box J2.

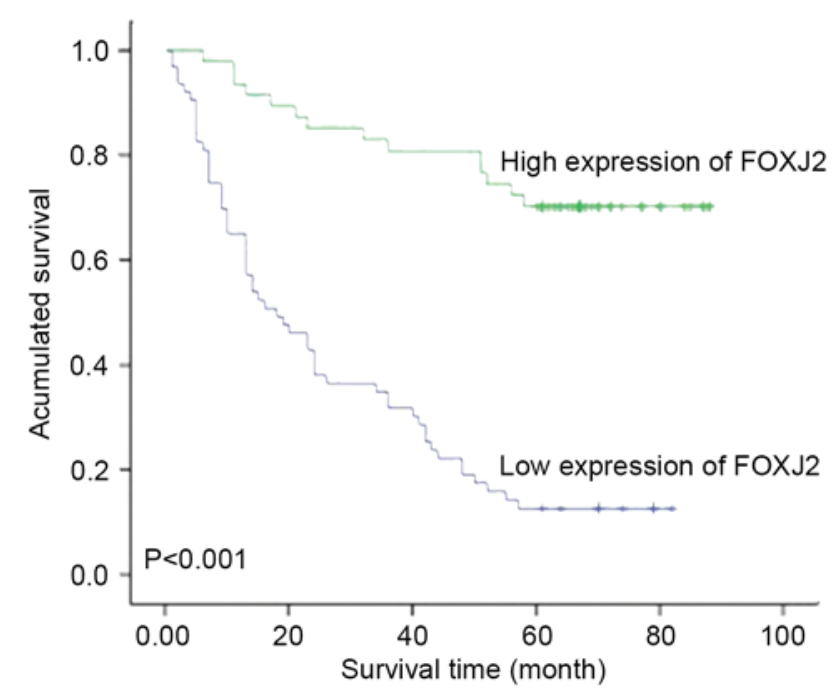

Figure 4. Kaplan-Meier survival curves for low vs. high FOXJ2 expression in 110 patients with hepatocellular carcinoma, exhibiting a significant separation between the curves $(\mathrm{P}<0.001, \log$-rank test). FOXJ2, forkhead box J2.

tion, and to develop novel targeted therapeutic strategies. Therefore, previous studies have focused on the development of therapeutic strategies based on molecular targeting in human cancer, including in HCC $(14,15)$. The current study demonstrated that reduced expression levels of FOXJ2 were significantly associated with the poor prognosis of patients with $\mathrm{HCC}$, which is partially attributed to the cell proliferation of HCC cells. Thus, it is suggested that the observations of the current study may benefit the development of novel therapeutic strategies for patients with HCC based on FOXJ2.

Members of the Fox gene family share an evolutionarily conserved DNA-binding domain, termed the 'fork-head' or 'winged-helix' domain $(16,17)$. The Fox family is a large and diverse group of transcription factors that serve various important
A
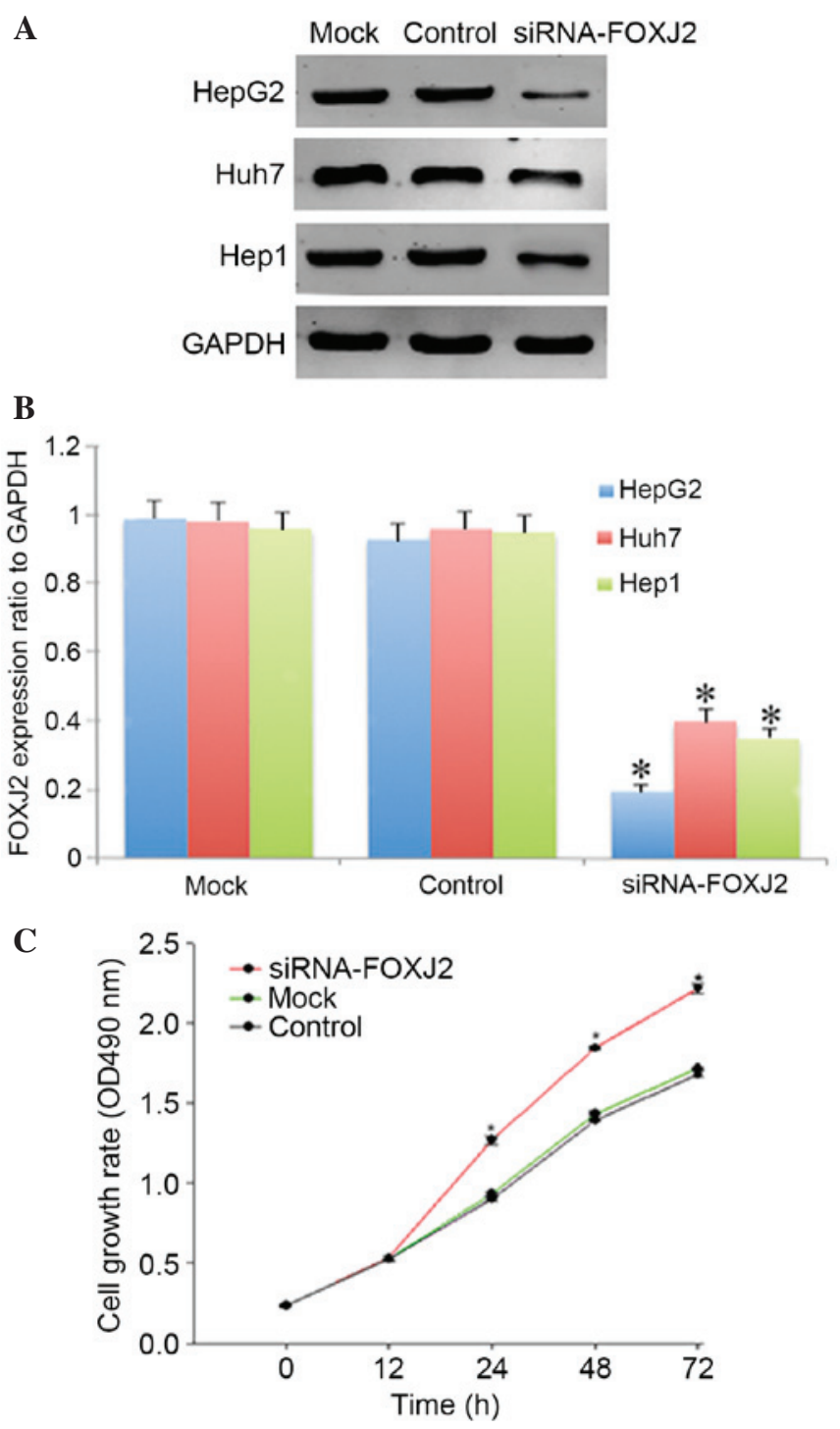

Figure 5. FOXJ2 knockdown promoted cell proliferation. (A) Western blot analysis indicated that siRNA treatment of FOXJ2 markedly reduced the FOXJ2 levels $48 \mathrm{~h}$ subsequent to siRNA transfection in three HCC cells in comparison with cells transfected with negative control and mock treatments. (B) The ratio of FOXJ2 protein to GAPDH expression measured by densitometry. Data are presented as the mean \pm standard error. (C) A CCK- 8 assay indicated that FOXJ2 knockdown in HepG2 cell promoted cell proliferation. CCK- 8 reagents were added to the medium and incubated for additional $2 \mathrm{~h}$. Absorbance was measured at each indicated time (0, 12, 24, 48 and $72 \mathrm{~h}$ ). Each time point was derived from three independent experiments. The data are presented as the mean \pm standard deviation for three experiments ${ }^{*} \mathrm{P}<0.05$ vs. mock and control groups. FOXJ2, forkhead box J2; siRNA, small interfering RNA; GAPDH, glyceraldehyde 3-phosphate dehydrogenase.

roles in a wide variety of biological processes including development, metabolism, immunology and senescence $(16,18,19)$. In addition, it has been reported that several members of this family are involved in the etiology of cancer $(20,21)$. The FOXA1 gene has been observed to be amplified and overexpressed in esophageal and lung cancer (22), and the FOXP1 transcription factor is suggested to function as either an oncogene or as a tumor suppressor that is cell type-dependent (23-26). Furthermore, FOXJ1 was observed to be markedly upregulated in human HCC specimens (27), and increased expression of FOXM1 protein was identified in variety of human tumors, including in HCC (28) and intrahepatic cholangiocarcinoma (29). 
FOXJ2 (FHX) is a recently characterized human forkhead transcriptional activator that binds DNA with a dual sequence specicity (30) and can be expressed as two isoforms, FHX.L and FHX.S, which differ in their C-terminal ends (6). In humans and mice, FOXJ2 expression can be detected in almost all tissues of the adult or the fetus. It has been identified to be localized constitutively at the nucleus of the cell and may be involved in the nuclear translocation mechanism of all forkhead factors. A previous study demonstrated that FOXJ2 served an important role in lipopolysaccharide-induced inflammatory responses, and that FOXJ2 may be involved in the activation of astrocytes (31). In addition, studies have demonstrated the association between FOXJ2 and cancer. For example, Wang et al (11) identified that the expression of FOXJ2 was higher in primary breast cancer tissues without lymph nodes metastases compared with those with, demonstrating that FOXJ2 can inhibit the metastasis of human breast cancer by regulating EMT key markers (E-cadherin and vimentin). Qiu et al (12) identified that FOXJ2 suppressed cell migration and invasion in glioma, and that overexpression of FOXJ2 increased E-cadherin expression and reduced vimentin expression, and significantly inhibited migration in U87 cells. Knockdown of FOXJ2 promoted cellular motility.

The current study identified that FOXJ2 may be an important prognotic factor in HCC. Western blotting and immunohistochemistry analysis identified that FOXJ2 was downregulated in HCC tissues and HCC cells, and observed that there was a significant negative correlation between FOXJ2 expression levels and HCC. FOXJ2 and Ki-67 were identified to be present predominantly in the nucleus, and FOXJ2 expression was negatively correlated with Ki67 expression. Accordingly, Kaplan-Meier survival analysis indicated that low expression of FOXJ2 was associated with poor prognosis of patients with HCC. Furthermore, it was demonstrated that FOXJ2 inhibited the proliferation of HCC using a CCK-8 assay. Knockdown of FOXJ2 expression was suggested to promote cell proliferation.

In summary, the results of the present study suggest that FOXJ2 is a novel and promising prognostic biomarker for HCC progression and prognosis. To the best of our knowledge, the current study is the first to investigate the clinical significance of FOXJ2 in HCC. With technological development, and using microarray analysis, numerous novel treatments may be developed based on the gene expression of tumors. The results of the current study may be useful in aiding in the prediction of prognosis, and may as a result be beneficial in the future treatment of patients with HCC.

\section{References}

1. Jemal A, Bray F, Center MM, Ferlay J, Ward E and Forman D: Global cancer statistics. CA Cancer J Clin 61: 69-90, 2011.

2. Altekruse SF, McGlynn KA and Reichman ME: Hepatocellular carcinoma incidence, mortality, and survival trends in the United States from 1975 to 2005. J Clin Oncol 27: 1485-1491, 2009.

3. Bruix J, Boix L, Sala M and Llovet JM: Focus on hepatocellular carcinoma. Cancer Cell 5: 215-219, 2004.

4. Lau WY and Lai EC: Hepatocellular carcinoma: Current management and recent advances. Hepatobiliary Pancreat Dis Int 7: 237-257, 2008.

5. Blum HE and Moradpour D: Viral pathogenesis of hepatocellular carcinoma. J Gastroenterol Hepatol 17 (Suppl 3): S413-S420, 2002 .
6. Pérez-Sánchez C, Gómez-Ferrería MA, de La Fuente CA, Granadino B, Velasco G, Esteban-Gamboa A and Rey-Campos J: FHX, a novel fork head factor with a dual DNA binding specificity. J Biol Chem 275: 12909-12916, 2000.

7. Pérez-Sánchez C, Arias-de-la-Fuente C, Gómez-Ferrería MA, Granadino B and Rey-Campos J: FHX.L and FHX.S, two isoforms of the human fork-head factor FHX (FOXJ2) with differential activity. J Mol Biol 301: 795-806, 2000.

8. Choi VM, Harland RM and Khokha MK: Developmental expression of FOXJ1.2, FOXJ2 and FOXQ1 in xenopus tropicalis. Gene Expr Patterns 6: 443-447, 2006.

9. Wijchers PJ, Hoekman MF, Burbach JP and Smidt MP: Identification of forkhead transcription factors in cortical and dopaminergic areas of the adult murine brain. Brain Res 1068: 23-33, 2006.

10. Kehn K, Berro R, Alhaj A, Bottazzi ME, Yeh WI, Klase Z, Van Duyne R, Fu S and Kashanchi F: Functional consequences of cyclin D1/BRCA1 interaction in breast cancer cells. Oncogene 26: 5060-5069, 2007.

11. Wang Y, Yang S, Ni Q, He S, Żhao Y, Yuan Q, Li C, Chen H, Zhang L, Zou L, et al: Overexpression of forkhead box J2 can decrease the migration of breast cancer cells. J Cell Biochem 113: 2729-2737, 2012.

12. Qiu X, Ji B, Yang L, Huang Q, Shi W, Ding Z, He X, Ban N, Fan S, Zhang J and Tian Y: The role of FOXJ2 in the migration of human glioma cells. Pathol Res Pract 211: 389-397, 2015.

13. Kudo M: Hepatocellular carcinoma 2009 and beyond: From the surveillance to molecular targeted therapy. Oncology 75 (Suppl 1): S1-S12, 2008.

14. Patel A and Sun W: Molecular targeted therapy in hepatocellular carcinoma: From biology to clinical practice and future. Curr Treat Options Oncol 15: 380-394, 2014.

15. Tsuchiya N, Sawada Y, Endo I, Saito K, Uemura Y and Nakatsura T: Biomarkers for the early diagnosis of hepatocellular carcinoma. World J Gastroenterol 21: 10573-10583, 2015.

16. Kaufmann E and Knöchel W: Five years on the wings of fork head. Mech Dev 57: 3-20, 1996.

17. Hannenhalli S and Kaestner KH: The evolution of fox genes and their role in development and disease. Nat Rev Genet 10: 233-240, 2009.

18. Carlsson P and Mahlapuu M: Forkhead transcription factors: Key players in development and metabolism. Dev Biol 250: $1-23,2002$

19. Jonsson H and Peng SL: Forkhead transcription factors in immunology. Cell Mol Life Sci 62: 397-409, 2005.

20. Benayoun BA, Caburet S and Veitia RA: Forkhead transcription factors: Key players in health and disease. Trends Genet 27: 224-232, 2011

21. Kaneda H, Arao T, Tanaka K, Tamura D, Aomatsu K, Kudo K, Sakai K, De Velasco MA, Matsumoto K, Fujita Y, et al: FOXQ1 is overexpressed in colorectal cancer and enhances tumorigenicity and tumor growth. Cancer Res 70: 2053-2063, 2010.

22. Lin L, Miller CT, Contreras JI, Prescott MS, Dagenais SL, Wu R, Yee J, Orringer MB, Misek DE, Hanash SM, et al: The hepatocyte nuclear factor 3 alpha gene, HNF3alpha (FOXA1), on chromosome band $14 \mathrm{q} 13$ is amplified and overexpressed in esophageal and lung adenocarcinomas. Cancer Res 62: 5273-5279, 2002

23. Banham AH, Connors JM, Brown PJ, Cordell JL, Ott G, Sreenivasan G, Farinha P, Horsman DE and Gascoyne RD: Expression of the FOXP1 transcription factor is strongly associated with inferior survival in patients with diffuse large B-cell lymphoma. Clin Cancer Res 11: 1065-1072, 2005.

24. Schuster MB and Porse BT: C/EBPalpha: A tumour suppressor in multiple tissues? Biochim Biophys Acta 1766: 88-103, 2006.

25. Barrans SL, Fenton JA, Banham A, Owen RG and Jack AS: Strong expression of FOXP1 identifies a distinct subset of diffuse large B-cell lymphoma (DLBCL) patients with poor outcome. Blood 104: 2933-2935, 2004.

26. Banham AH, Beasley N, Campo E, Fernandez PL, Fidler C, Gatter K, Jones M, Mason DY, Prime JE, Trougouboff P, et al: The FOXP1 winged helix transcription factor is a novel candidate tumor suppressor gene on chromosome $3 \mathrm{p}$. Cancer Res 61: 8820-8829, 2001.

27. Chen HW, Huang XD, Li HC, He S, Ni RZ, Chen CH, Peng C, Wu G, Wang GH, Wang YY, et al: Expression of FOXJ1 in hepatocellular carcinoma: Correlation with patients prognosis and tumor cell proliferation. Mol Carcinog 52: 647-659, 2013 
28. Lee JS, Chu IS, Heo J, Calvisi DF, Sun Z, Roskams T, Durnez A, Demetris AJ and Thorgeirsson SS: Classification and prediction of survival in hepatocellular carcinoma by gene expression profiling. Hepatology 40: 667-676, 2004.

29. Obama K, Ura K, Li M, Katagiri T, Tsunoda T, Nomura A Satoh S, Nakamura Y and Furukawa Y: Genome-wide analysis of gene expression in human intrahepatic cholangiocarcinoma. Hepatology 41: 1339-1348, 2005.
30. Granadino B, Arias-de-la-Fuente C, Pérez-Sánchez C, Párraga M, López-Fernández LA, del Mazo J and Rey-Campos J: FHX (FOXJ2) expression is activated during spermatogenesis and very early in embryonic development. Mech Dev 97: 157-160, 2000.

31. Chen X, Cao X, Tao G, Cao Z, Wang S, Zhou F, Xie W, Zhao P, Zhang $\mathrm{Z}$ and Cui Z: FOXJ2 expression in rat spinal cord after injury and its role in inflammation. J Mol Neurosci 47: 158-165, 2012. 